\title{
Hardware-in-the-loop simulation for a sliding mode FDI scheme
}

\author{
Lejun Chen $^{1}$, Christopher Edwards ${ }^{1}$, Halim Alwi ${ }^{1}$ and Masayuki Sato ${ }^{2}$
}

\begin{abstract}
This paper describes the implementation of a sliding mode observer based fault detection and isolation (FDI) scheme, which is capable of reconstructing/estimating sensor faults, on the Japan Aerospace Exploration Agency's Multi-Purpose Aviation Laboratory (MuPAL- $\alpha$ ) research aircraft. Hardware-in-the-loop (HIL) simulation results, which serve as a precursor to upcoming real test flights, are presented in this paper. The HIL simulation results show good yaw rate sensor fault reconstruction performance, and will be flight tested as the next stage of development and validation.
\end{abstract}

\section{INTRODUCTION}

Modern model-based fault detection and isolation (FDI) methods, also referred to as analytic redundancy, represent one possible approach to health monitoring for aircraft (see for example the results from GARTEUR FM-AG16 [1]). Furthermore, analytic redundancy FDI approaches can improve the sustainability of aircraft, by helping to reduce over-engineering and replacing hardware redundancy based fault detection logic. Consequently the weight of the aircraft, fuel consumption and design complexity can be reduced.

The most recent large scale funded investigation into the potential of fault detection and isolation/fault tolerant control (FDI/FTC) for aircraft flight control systems is the H2020/Japan co-funded project VISION (Validation of Integrated Safety-enhanced Intelligent Flight cONtrol). The aim of the project is to develop and validate in-flight FDI/FTC techniques for aircraft guidance, navigation and control, with the objective of increasing TRL levels to 7. One of the platforms to be used for flight testing is the Japan Aerospace Exploration Agency's MuPAL- $\alpha$ [2].

As a precursor to flight tests, this paper describes recent Hardware-in-the-loop (HIL) tests of a sliding mode observer (SMO) for sensor fault reconstruction. Sliding mode observers (SMOs) are capable of reconstructing unmeasurable signals within a process by appropriate filtering of the 'equivalent output error injection' signal required to maintain sliding [3]. This is a unique property of sliding mode observers, which emanates from the fact that the introduction of a sliding motion forces the outputs of the observer to perfectly track the plant measurements [3]. Theoretically, sliding mode observers can provide finite time convergence of the state estimation errors to zero and totally reject the effects of a class of unknown bounded matched disturbances. This property of total rejection is quite different from the characteristics of high gain observers [4] which only provide ultimate boundedness guarantees; or $\mathcal{H}_{\infty}$ filters [5] in which the effect of disturbances are optimally attenuated by the appropriate choice of observer gain, but are not usually totally rejected. Furthermore sliding mode observers have been shown to perform well when directly compared with other methods [6], [7] for different types of system. The ability to accurately reconstruct faults is useful since the estimates can be used to create (virtual) corrected measurements for use by the control law (i.e. FTC) [8]. Other methods including $\mathcal{H}_{\infty}$ and adapting schemes are being developed within the VISION project and those results are reported elsewhere [9], [10]. This paper focusses on the design of an SMO for sensor fault estimation. The proposed scheme is capable of reconstructing sensor faults in the presence of external disturbances.

In this paper, an SMO based FDI scheme has been developed and implemented within MuPAL- $\alpha$ 's HIL test platform, as a precursor to actual flight tests. The HIL setup includes the real aircraft, with all its attendant systems, connected to a computer to provide a realistic emulated environment.

This paper focusses on the lateral-directional dynamics. The scenarios considered concern yaw rate sensor faults. Since the sensor fault is reconstructed, the scheme has the potential to achieve fault tolerant control without reconfiguring the baseline controller (however this aspect is not pursued in this particular publication). The main contribution of the paper is, as far as the authors are aware, the first implementation and validation of an SMO based FDI scheme at a system integration level within an on-board fly-by-wire system, in which a real aircraft is involved in hardware-in-the-loop ground testing.

\footnotetext{
*VISION project: Supported by EU-H2020 (690811) and Japan-NEDO (062800) grants

${ }^{1}$ College of Engineering, Mathematics and Physical Sciences, University of Exeter, UK. 1 c 427, h. a lwi, c. edwards@ex.ac.uk ${ }^{2}$ Japan Aerospace Exploration Agency, Mitaka, Tokyo 181-0015, Japan. sato.masayuki @ jaxa.jp
} 
The structure of the paper is as follows: Section II describes the SMO based FDI scheme. In Section III, the effectiveness of the scheme is tested on the HIL test platform. The corresponding design and validation results are then presented. Section IV concludes the paper and outlines the plans for future work - including piloted in-flight testing.

\section{SLIDING MODE OBSERVER}

This section begins by recapitulating the approach for sensor fault reconstruction proposed in [11]. Consider an uncertain linear time invariant (LTI) system subject to sensor faults described by

$$
\begin{aligned}
\dot{x}_{p}(t) & =A_{p} x_{p}(t)+B_{p} u_{p}(t)+M_{p} \xi(t) \\
y_{p}(t) & =C_{p} x_{p}(t)+H_{p} f(t)
\end{aligned}
$$

where, $A_{p} \in \mathbb{R}^{n \times n}, B_{p} \in \mathbb{R}^{n \times m}, C_{p} \in \mathbb{R}^{p \times n}, M_{p} \in \mathbb{R}^{n \times l}$, and $H_{p} \in \mathbb{R}^{p \times q}$ where $q<p$. In (1), $H_{p}$ represents the sensor fault distribution matrix, and in this paper the columns of $H_{p}$ are assumed to belong to the standard basis for $\mathbb{R}^{p}$ i.e. the column of $H_{p}$ are formed from ones and zeros, and in each column exactly one element is unity [12]. It is assumed $C_{p}$ is full row rank and $H_{p}$ is full column rank. In (1) it is assumed that $u_{p}(t)$ and $y_{p}(t)$ are measurable, $x_{p}(t)$ is unknown, and that $y_{p}(t)$ represents the sensor measurements potentially corrupted by faults (represented by $f(t)$ ). The signal $f(t)$ is unknown, but subject to

$$
\|f(t)\| \leq \beta(t)
$$

where $\beta(t)$ is a known function. The signal $\xi(t)$ denotes a bounded external disturbance and is assumed to satisfy

$$
\|\xi(t)\| \leq d
$$

where $d$ is known. Since $H_{p}$ is composed of columns from the standard basis for $\mathbb{R}^{p}$, by permutating the elements of $y_{p}$, it is easy to obtain the form

$$
\left[\begin{array}{l}
y_{p, 1}(t) \\
y_{p, 2}(t)
\end{array}\right]=\left[\begin{array}{l}
C_{1} \\
C_{2}
\end{array}\right] x_{p}(t)+\left[\begin{array}{c}
0 \\
I_{q}
\end{array}\right] f(t)
$$

where $C_{1} \in \mathbb{R}^{(p-q) \times n}$ and $C_{2} \in \mathbb{R}^{q \times n}$, and $y_{p, 2}(t)$ denotes the outputs potentially corrupted by sensor faults.

The objective is to design an SMO to estimate the unknown states and $f(t)$. To reformulate the sensor fault reconstruction problem as a more general actuator fault reconstruction scheme, as in [11], introduce a (stable) filter in the form of

$$
\dot{z}_{f}(t)=-A_{f} z_{f}(t)+A_{f} y_{p, 2}(t)
$$

where $z_{f}(t) \in \mathbb{R}^{q}$ and the matrix $A_{f}$ is a symmetric positive definite (s.p.d) matrix. Combining (1), (4) and (5), the augmented system can be conveniently represented by

$$
\begin{aligned}
{\left[\begin{array}{c}
\dot{x}_{p}(t) \\
\dot{z}_{f}(t)
\end{array}\right] } & =\left[\begin{array}{cc}
A_{p} & 0 \\
A_{f} C_{2} & -A_{f}
\end{array}\right]\left[\begin{array}{l}
x_{p}(t) \\
z_{f}(t)
\end{array}\right]+\left[\begin{array}{c}
B_{p} \\
0
\end{array}\right] u_{p}(t)+\left[\begin{array}{c}
0 \\
A_{f}
\end{array}\right] f(t)+\left[\begin{array}{c}
M_{p} \\
0
\end{array}\right] \xi(\cdot) \\
{\left[\begin{array}{c}
y_{p, 1}(t) \\
z_{f}(t)
\end{array}\right] } & =\left[\begin{array}{cc}
C_{1} & 0 \\
0 & I_{q}
\end{array}\right]\left[\begin{array}{l}
x_{p}(t) \\
z_{f}(t)
\end{array}\right]
\end{aligned}
$$

Remark 2.1: Note that the choice of the filter gain $A_{f}$ affects the system performance and as such constitutes an important design parameter. For more details see the discussion on page 216 in [11].

Define a state coordinate transformation matrix for (6) as

$$
T_{a}=\operatorname{Diag}\left\{T_{s}, I_{q}\right\}
$$

where the square nonsingular matrix $T_{s} \in \mathbb{R}^{n \times n}$ is any matrix with the property that $C_{1} T_{s}^{-1}=\left[\begin{array}{ll}0 & I_{p-q}\end{array}\right]$. Note that since by assumption $C_{p}$ is full row rank, $C_{1}$ has full row rank. Applying the above coordinate transformation to (6) yields the representation

$$
\underbrace{\left[\begin{array}{c}
\dot{x}(t) \\
\dot{z}_{f}(t)
\end{array}\right]}_{\dot{x}_{a}(t)}=\underbrace{\left[\begin{array}{cc}
T_{s} A_{p} T_{s}^{-1} & 0 \\
A_{f} C_{2} T_{s}^{-1} & -A_{f}
\end{array}\right]}_{A} \underbrace{\left[\begin{array}{c}
x(t) \\
z_{f}(t)
\end{array}\right]}_{x_{a}(t)}+\underbrace{\left[\begin{array}{c}
T_{s} B_{p} \\
0
\end{array}\right]}_{B} u_{p}(t)+\underbrace{\left[\begin{array}{c}
0 \\
A_{f}
\end{array}\right]}_{D} f(t)+\underbrace{\left[\begin{array}{c}
T_{s} M_{p} \\
0
\end{array}\right]}_{M} \xi(\cdot)
$$

In this coordinate system, $y_{a}=\operatorname{Col}\left(y_{p, 1}, z_{f}\right)=C x_{a}$, where

$$
C=\left[\begin{array}{cc}
C_{1} T_{s}^{-1} & 0 \\
0 & I_{q}
\end{array}\right]=\left[\begin{array}{ll}
0 & I_{p}
\end{array}\right]
$$




\section{A. Observer Structure}

The system $(A, B, C)$ from (8) with fault distribution matrix $D$ and with disturbance distribution matrix $M$ from (8)-(9) will be used as the basis of the observer design.

A classical/conventional SMO will be used here which requires relative degree one minimum phase conditions [11]. The rank condition on the Markov is trivially satisfied since $C D=\left[\begin{array}{ll}0 & A_{f}^{T}\end{array}\right]^{T}$ where $\operatorname{rank}\left(A_{f}\right)=q$ since it is s.p.d by design. The minimum phase condition is more subtle, but a sufficient condition is that the system matrix $A_{p}$ is Hurwitz [11]. The most straightforward test for applicability is to check the invariant zeros of $(A, D, C)$ lies in the LHP. In the example which will be considered in Section III, this condition is fulfilled. It can be shown from [11] that an appropriate SMO to address this problem is one with the structure

$$
\dot{z}(t)=A z(t)+B u_{p}(t)+G_{l} e_{y}(t)+G_{n} \nu(t)
$$

where the output estimation error $e_{y}(t)=C\left(z(t)-x_{a}(t)\right)$ and $\nu(t)$ represents the discontinuous output error injection vector used to induce the sliding motion. Here

$$
\nu=\left\{\begin{array}{cc}
-\rho(t) \frac{e_{y}}{\left\|e_{y}\right\|} & \text { if } e_{y} \neq 0 \\
0 & \text { otherwise }
\end{array}\right.
$$

where the modulation gain $\rho(t)$ will be specified later. The matrix $G_{n} \in \mathbb{R}^{(n+q) \times p}$ in (10) is given by

$$
G_{n}=\left[\begin{array}{c}
-L \\
I_{p}
\end{array}\right]
$$

where in turn the design parameter $L \in \mathbb{R}^{(n+q-p) \times p}$ has the structure

$$
L=\left[\begin{array}{ll}
L_{1} & 0
\end{array}\right]
$$

and the matrix $L_{1} \in \mathbb{R}^{(n+q-p) \times(p-q)}$ [11]. Suppose the state estimation error $e=z-x_{a}=\operatorname{col}\left(e_{1}, e_{y}\right)$, where, $e_{1} \in \mathbb{R}^{n+q-p}$, then it follows

$$
\left[\begin{array}{c}
\dot{e}_{1} \\
\dot{e}_{y}
\end{array}\right]=\left[\begin{array}{ll}
A_{11} & A_{12} \\
A_{21} & A_{22}
\end{array}\right]\left[\begin{array}{c}
e_{1} \\
e_{y}
\end{array}\right]-\left[\begin{array}{c}
0 \\
D_{2}
\end{array}\right] f-\left[\begin{array}{c}
M_{1} \\
M_{2}
\end{array}\right] \xi+\left[\begin{array}{l}
G_{l 1} \\
G_{l 2}
\end{array}\right] e_{y}+\left[\begin{array}{c}
-L \\
I_{p}
\end{array}\right] \nu
$$

where $A_{11} \in \mathbb{R}^{(n+q-p) \times(n+q-p)}, D_{2} \in \mathbb{R}^{p \times q}$ and $M_{2} \in \mathbb{R}^{p \times l}$ and are given by

$$
D_{2}=\left[\begin{array}{c}
0 \\
A_{f}
\end{array}\right], \quad M_{2}=\left[\begin{array}{c}
M_{21} \\
0
\end{array}\right]
$$

where $M_{21} \in \mathbb{R}^{(p-q) \times q}$. These special structures arise from the specific augmentation in (6). Define a coordinate transformation $e \mapsto \tilde{e}=T_{L} e$, given by

$$
T_{L}=\left[\begin{array}{cc}
I_{n+q-p} & L \\
0 & I_{p}
\end{array}\right]
$$

Applying the above transformation to (14), in the new coordinates, the error system is represented by

$$
\left[\begin{array}{c}
\dot{\tilde{e}}_{1} \\
\dot{e}_{y}
\end{array}\right]=\left[\begin{array}{cc}
\tilde{A}_{11} & \tilde{A}_{12} \\
A_{21} & \tilde{A}_{22}
\end{array}\right]\left[\begin{array}{c}
\tilde{e}_{1} \\
e_{y}
\end{array}\right]-\left[\begin{array}{c}
0 \\
D_{2}
\end{array}\right] f-\left[\begin{array}{c}
\tilde{M}_{1} \\
M_{2}
\end{array}\right] \xi+\left[\begin{array}{c}
\tilde{G}_{l 1} \\
G_{l 2}
\end{array}\right] e_{y}+\left[\begin{array}{c}
0 \\
I_{p}
\end{array}\right] \nu
$$

where $\tilde{A}_{11}=A_{11}+L A_{21}, \tilde{M}_{1}=M_{1}+L M_{2}, \tilde{e}_{1}=e_{1}+L e_{y}$ and $\tilde{e}=\operatorname{Col}\left(\tilde{e}_{1}, e_{y}\right)$. The structure of the fault distribution matrix in (17) occurs because $L D_{2}=0$. This occurs as a consequence of the special form of $L_{1}$ in (13) and $D_{2}$ in (15) [11]. In this error system define

$$
\left[\begin{array}{l}
\tilde{G}_{l 1} \\
G_{l 2}
\end{array}\right]=\left[\begin{array}{c}
-\tilde{A}_{12} \\
-\tilde{A}_{22}-k_{2} I_{p}
\end{array}\right]
$$

where $k_{2}$ is a positive scalar to be designed.

The remainder of this section (focussing on observer design) is bespoke to this paper and represents developments beyond those in [11].

Notice from (17) and (18) that

$$
\dot{\tilde{e}}_{1}(t)=\tilde{A}_{11} \tilde{e}_{1}(t)-\tilde{M}_{1} \xi(t)
$$


where $\tilde{A}_{11}$ is assumed to be Hurwitz (by selecting a suitable $L$ ). Since $\tilde{A}_{11}$ is Hurwitz

$$
\left\|e^{\tilde{A}_{11} t}\right\| \leq m e^{-\alpha_{0} t}
$$

for some positive scalars $m$ and $\alpha_{0}$ (from the semi-group property of linear systems). Therefore

$$
\begin{aligned}
\left\|\tilde{e}_{1}(t)\right\| & \leq m e^{-\alpha_{0} t}\left\|\tilde{e}_{1}(0)\right\|+\int_{0}^{t} m\left\|\tilde{M}_{1}\right\| e^{-\alpha_{0}(t-s)}\|\xi(s)\| d s \\
& \leq m e^{-\alpha_{0} t}\left\|\tilde{e}_{1}(0)\right\|+d m\left\|\tilde{M}_{1}\right\| / \alpha_{0}
\end{aligned}
$$

since from (3), by assumption $\|\xi(t)\| \leq d$. Therefore for an appropriately chosen scalar $\beta_{0}>0$

$$
\left\|\tilde{e}_{1}(t)\right\| \leq \beta_{0}+d m\left\|\tilde{M}_{1}\right\| / \alpha_{0}
$$

in finite time $t_{0}$ where $t_{0} \leq-\frac{1}{\alpha_{0}} \log _{e}\left(m\left\|\tilde{e}_{1}(0)\right\| / \beta_{0}\right)$.

Theorem 2.1: If the modulation gain from (11) satisfies

$$
\rho(t) \geq\left\|A_{21}\right\|\left(\beta_{0}+d m\left\|\tilde{M}_{1}\right\| / \alpha_{0}\right)+\left\|D_{2}\right\| \beta(t)+\left\|M_{2}\right\| d+\eta
$$

then a sliding motion on $\mathcal{S}=\left\{\tilde{e} \in \mathbb{R}^{n+q}: C \tilde{e}=0\right\}$ can be enforced in finite time.

Proof: Consider a candidate Lyapunov function $V\left(e_{y}\right)=\frac{1}{2} e_{y}^{T} e_{y}$. For all $t>t_{0}$, the derivative along the trajectory is

$$
\begin{aligned}
\dot{V} & =e_{y}^{T}\left(A_{21} \tilde{e}_{1}+\tilde{A}_{22} e_{y}-D_{2} f-M_{2} \xi+\tilde{G}_{l 2} e_{y}+\nu\right) \\
& =e_{y}^{T}\left(A_{21} \tilde{e}_{1}-k_{2} e_{y}-D_{2} f-M_{2} \xi+\nu\right) \\
& \leq\left\|e_{y}\right\|\left(\left\|A_{21}\right\|\left\|\tilde{e}_{1}\right\|+\left\|D_{2}\right\| \beta(t)+\left\|M_{2}\right\| d-\rho(t)\right) \\
& \leq-\eta\left\|e_{y}\right\|=-\eta \sqrt{2 V}
\end{aligned}
$$

This ensures sliding occurs in finite time [13].

\section{B. Fault reconstruction}

During sliding, $\dot{e}_{y}(t)=e_{y}(t)=0$ [13]. Then substituting these quantities into (17) yields the expressions

$$
\begin{aligned}
\dot{\tilde{e}}_{1} & =\tilde{A}_{11} \tilde{e}_{1}-\left(M_{1}+L M_{2}\right) \xi \\
0 & =A_{21} \tilde{e}_{1}-D_{2} f-M_{2} \xi+\nu_{e q}
\end{aligned}
$$

where $\nu_{e q}$ is the equivalent output error injection signal ([13]). In this paper, consider a fault estimation signal

$$
\hat{f}=W \nu_{e q}
$$

In equation (26) the user defined gain

$$
W=\left[\begin{array}{ll}
W_{1} & A_{f}^{-1}
\end{array}\right]
$$

where $W_{1} \in \mathbb{R}^{q \times(p-q)}$ denotes the design freedom. Define $e_{f}=\hat{f}-f$, then it follows from (25) and (26)

$$
e_{f}=-W A_{21} \tilde{e}_{1}+W D_{2} f+W M_{2} \xi-f
$$

Let

$$
A_{21}=\left[\begin{array}{ll}
A_{211}^{T} & A_{212}^{T}
\end{array}\right]^{T}
$$

Then using the fact that $W D_{2}=I, W M_{2}=W_{1} M_{21}$ and $W A_{21}=W_{1} A_{211}+A_{f}^{-1} A_{212}$, the observer error dynamics and the fault estimation error satisfies

$$
\begin{aligned}
& \dot{\tilde{e}}_{1}=\tilde{A}_{11} \tilde{e}_{1}-\tilde{M}_{1} \xi \\
& e_{f}=\left(-W_{1} A_{211}-A_{f}^{-1} A_{212}\right) \tilde{e}_{1}+W_{1} M_{21} \xi
\end{aligned}
$$

Theorem 2.2: An upper bound on the $\mathcal{L}_{2}$ gain $\gamma$ for the system in (30) can be computed so that

$$
\left\|e_{f}\right\|_{2} \leq \gamma\|\xi\|_{2}
$$


if there exists a s.p.d matrix $P_{0}$ and design freedom $Y$ and $W_{1}$ such that

$$
\left[\begin{array}{ccc}
\operatorname{He}\{\cdot\} & -P_{0} M_{1}-Y M_{2} & \left(-W_{1} A_{211}-A_{f}^{-1} A_{212}\right)^{T} \\
* & -\gamma I & \left(W_{1} M_{21}\right)^{T} \\
* & * & -\gamma I
\end{array}\right]<0
$$

where $\operatorname{He}\{\cdot\}=\operatorname{He}\left\{P_{0} A_{11}+Y A_{21}\right\}$.

Proof: From the Bounded Real Lemma, (31) is guaranteed if (32) holds. It follows (32) is an LMI with respect to the decision variables $P_{0}, Y$ and $W_{1}$. By solving the LMI in (32), the observer gain $L=P_{0}^{-1} Y$.

The observer discussed in this section will now be applied to the problem of reconstruction of a yaw rate sensor fault associated with the MuPAL- $\alpha$ aircraft.

\section{HIL TEST ON THE MUPAL- $\alpha$ PLATFORM}

\section{A. MuPAL- $\alpha$ platform}

MuPAL- $\alpha$ is owned and operated by the Japan Aerospace Exploration Agency. The aircraft is a Dornier Do228202 modified to include a bespoke Fly-By-Wire (FBW) system. The MuPAL- $\alpha$ platform (see Fig. 1) is used for both Hardware-in-the-loop (HIL) ground tests and piloted flight tests of new guidance and control algorithms (and the associated human factors). Researchers are able to incorporate new control laws or test flight displays for

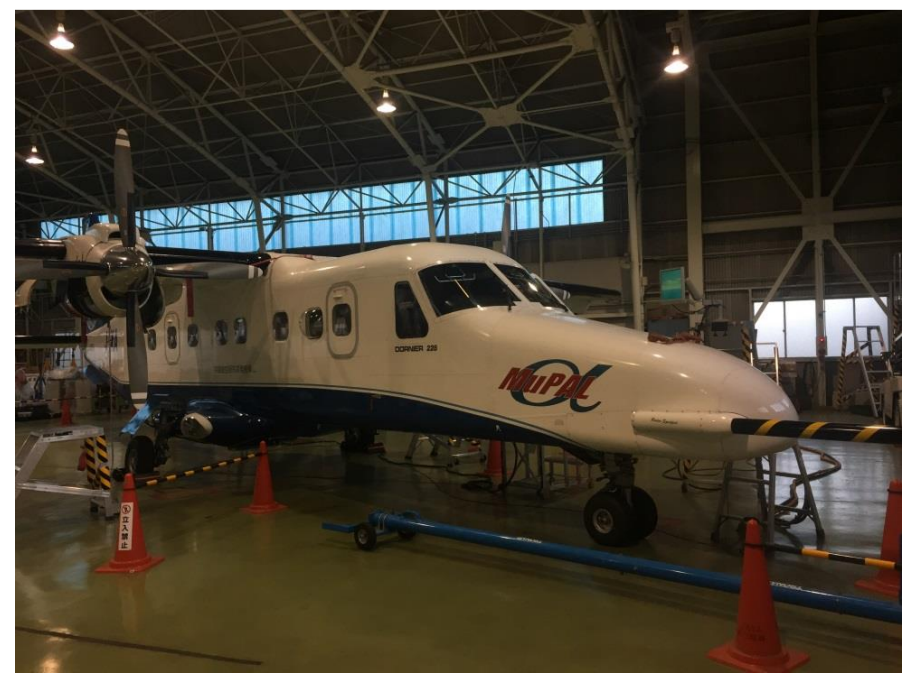

Fig. 1. MuPAL- $\alpha$ HIL test platform

the pilot. MuPAL- $\alpha$ can be used to simulate the motion of various types of aircraft (big transports, business jets etc), and also to test the effect of system failures (see for example [14], [15], [16], [17], [18]). From a safety perspective, the primary elements of the bespoke on-board FBW system are designed to be duplex, allowing a safety pilot to override the FBW output and manually take over control via the original mechanical control system [2]. Consequently, MuPAL- $\alpha$ represents a unique facility for flight testing FDI and FTC schemes.

\section{B. MuPAL- $\alpha$ HIL test platform}

Following JAXA's test procedures, HIL simulations are always carried out first to test the C-code implementations of any new FDI or FTC schemes, to provide a preliminary assessment of their performance before any actual piloted test flights. The HIL configuration allows a wide range of manoeuvres and flight conditions to be conducted in safe static conditions inside the hanger. The HIL test setup has the capability to include various wind and gust conditions to mimic actual flight conditions as a further check. The HIL tests allow the safety and evaluation pilots to become acquainted with the manoeuvres and fault scenarios to be conducted during the actual flight tests.

The MuPAL- $\alpha$ HIL test platform represents a fairly conventional flight simulator arrangement, but includes the actual aircraft as part of the configuration. An emulation computer provides the flight simulator capabilities and runs in real time a high fidelity simulation model of the aircraft which has been calibrated against real flight data. 
The emulation computer uses the position of the actual aircraft's control surfaces (which respond during the HIL tests) as inputs to the simulation. The simulation on the emulation computer then provides 'sensor measurements' of the position and attitude of the aircraft. These emulated measurements provide the signals which are used by the control law running on the FBW computer, and update the cockpit displays for the pilots. The emulation computer also updates the graphic display screen (placed outside of the cockpit) to provide the pilots with a visualisation of the aircraft attitude. In this way the FDI and FTC interact with the real system hardware on the aircraft. For further details of MuPAL- $\alpha$ and its operation, see for example [2].

\section{Hardware-in-the-loop tests}

This paper focuses on the lateral-directional dynamics of the aircraft. The results which will be presented in the following subsections were all obtained from HIL tests. A control scheme was initially coded in C and integrated into the FBW system. The controller which is used is the sliding mode control allocation scheme described in [19]. During the HIL tests, it is assumed that the yaw rate sensor measurement is corrupted by faults (which are introduced at a software level). All the faults are assumed to occur from 30sec onwards. Since FTC is not explored in this paper, during the HIL tests illustrated in this paper, the sensor faults are not fed back to the baseline controller taken from [19], which has good sideslip and roll angle tracking performance, and therefore the closed-loop system performance is always fault-free. The system architecture for the HIL implementation is shown in Fig. 2.

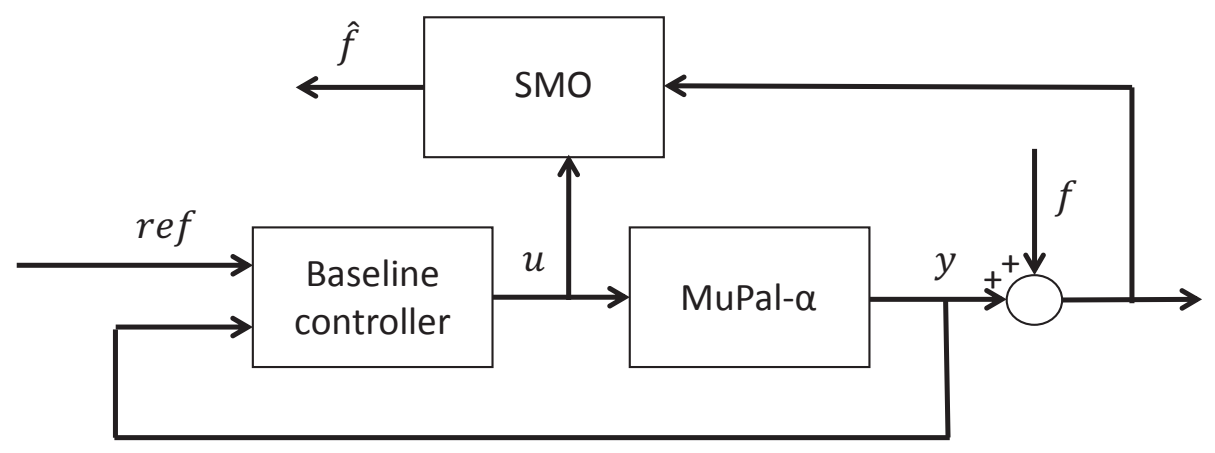

Fig. 2. MuPAL- $\alpha$ HIL test

During the HIL tests, the sideslip and roll angle commands are created by the evaluation pilot via the pedal and wheel respectively.

\section{Observer design results}

During the HIL tests, the flaps are retracted. The Direct Lift Control (DLC) flaps are fixed at 0 deg while the gear (which is used to set MuPAL- $\alpha$ on the ground) is set as retracted in the simulation. The weight of the aircraft is $5700 \mathrm{~kg}$, the centre of gravity is set at $28 \%$, the trim altitude is $5000 \mathrm{ft}$ and the trim indicated airspeed is 120 kts. A lateral-directional LTI model of MuPAL- $\alpha$ at this specific flight condition was provided by the Japan Aerospace Exploration Agency (JAXA) based on recorded flight test data.

The system states of the LTI model are given by

$$
x_{p}=\left[\begin{array}{llll}
\phi & \beta & p & r
\end{array}\right]^{T}
$$

which denote roll angle, sideslip angle, roll rate and yaw rate, respectively. In this paper, it is assumed that all system states are available and therefore $y_{p}=x_{p}$. The system inputs $u_{p}$ are given by

$$
u_{p}=\left[\begin{array}{ll}
\delta_{a} & \delta_{r}
\end{array}\right]^{T}
$$

where $\delta_{a}$ and $\delta_{r}$ represent the aileron and rudder surface deflections. Consequently, in this example, $n=4, p=4$ and $q=1$ and

$$
C_{p}=\left[\frac{C_{p, 1}}{C_{p, 2}}\right]=\left[\begin{array}{cccc}
1 & 0 & 0 & 0 \\
0 & 1 & 0 & 0 \\
0 & 0 & 1 & 0 \\
\hline 0 & 0 & 0 & 1
\end{array}\right], \quad H_{p}=\left[\begin{array}{l}
0 \\
0 \\
0 \\
1
\end{array}\right]
$$


In this scenario, the disturbance distribution matrix is

$$
M_{p}=\left[\begin{array}{cccc}
10 & 0 & 0 & 0 \\
0 & 0 & 0 & 1
\end{array}\right]^{T}
$$

This matrix is used as (indirect) design freedom for the synthesis of $L$. In this paper, by considering that $\xi(t)$ represents the external disturbance and roll signal is not so often corrupted by noise compared to yaw rate signal, the roll angle signal is supposed to be affected by ten-times large external disturbance compared to yaw rate signal, i.e. roll angle signal accept large external disturbance. The filter parameter has been chosen as $A_{f}=0.01$. By choosing a suitable gain matrix $L$ for (12) and (13), the matrix $\tilde{A}_{11}$ is established. In this design, $k_{2}=0.1$, and the values of $L$ and $W_{1}$ obtained using the Matlab LMI toolbox are

$$
\begin{aligned}
L & =\left[\begin{array}{llll}
0.6123 & 4.7950 & -1.2778 & 0
\end{array}\right] \\
W_{1} & =\left[\begin{array}{lll}
0.0055 & 0.9216 & -0.0576
\end{array}\right]
\end{aligned}
$$

The modulation gain $\rho(t)$ is chosen as 0.7 , and the small positive scalar selected for the sigmoidal approximation [13] to the discontinuous injection term is 0.01 and the expression in (11) is then replaced by

$$
\nu=-\rho(t) \frac{e_{y}}{\left\|e_{y}\right\|+0.01}
$$

\section{E. HIL simulation results}

In this section, the HIL simulation results, associated with additive sine wave faults, are illustrated.

1) Fault free without wind affects: The manoeuvre shown in Fig. 3(a) is manually created by the evaluation pilot via wheel manipulation and represents a roll angle command of approximately $20 \mathrm{deg}$ to $-20 \mathrm{deg}$. Fig. 3(b) shows the observer performance in the absence of faults and without any wind/gust disturbances in the emulation tests. It can be seen from Fig. 3(b) that the fault reconstruction signal is small. The sliding surface variable $\left\|e_{y}\right\|$ is zero from the beginning of the simulation. It can be seen from Fig. 3(b) that there exist small spikes on $\left\|e_{y}\right\|$ corresponding to the tunes of the pilot manipulations. The emulated wind, injected during the HIL simulation, is almost zero. The fault estimation error, at the sample times, plotted as a histogram, displays a tight normal distribution with a small nonzero mean - indicating a small bias (approx $0.5 \mathrm{deg} / \mathrm{s}$ ).

2) Faulty cases: The HIL simulation results, associated with an additive sine wave yaw rate sensor fault, are shown in Fig. 5. The amplitude of the sine wave fault is $5 \mathrm{deg} / \mathrm{s}$ and the frequency of the sine wave fault is 0.05 Hz. A similar manoeuvre (as shown in Fig. 5(a)) is manually created by the evaluation pilot via pedal and wheel manipulations. Figure 5(b) shows the observer performance in the presence of the fault and the wind injected into the HIL system from a Dryden model (as shown in Fig. 4). It can be seen from Fig. 5(b) that the nominal yaw rate sensor measurement is affected by the sine wave fault from 30sec onwards. The observer is capable of reconstructing the sine wave fault to an accuracy of about 1deg/s as shown in the histogram of the fault estimation error (which has a larger standard deviation compared to the wind free case) and the fault reconstruction error is similar to the fault free case. The sliding variable $\left\|e_{y}\right\|$ is close to zero despite the fault and the wind.

These results are very satisfactory and are of sufficient interest that in future work piloted flight tests will take place.

\section{CONCLUSION}

This paper has shown the results of using a sliding mode observer based sensor FDI scheme to reconstruct/estimate yaw rate sensor faults in the presence of wind disturbances. The proposed scheme has been implemented on the Japan Aerospace Exploration Agency MuPAL- $\alpha$ research aircraft, and validated by ground based Hardware-in-theLoop (HIL) tests. The illustrated HIL results show that, during a steady turn manoeuvre induced by the evaluation pilot, yaw rate sensor faults can be well reconstructed to an accuracy of approximately $1 \mathrm{deg} / \mathrm{sec}$. These results are a precursor to planned actual piloted flight tests, and the HIL tests shown in this paper have yielded sufficiently good results to make these flight testes possible. The FDI scheme is intended to form part of a sensor fault tolerant flight control system obtained by using the fault estimates to correct the measured signals resulting in a virtual (fault-free) sensor reading. Flight tests of such an FTC scheme are also scheduled to take place and will be reported on during the conference if available. 

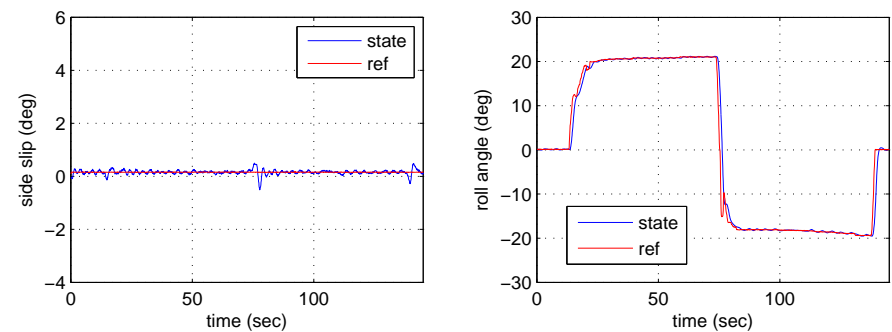

(a) Commands created by the evaluation pilot
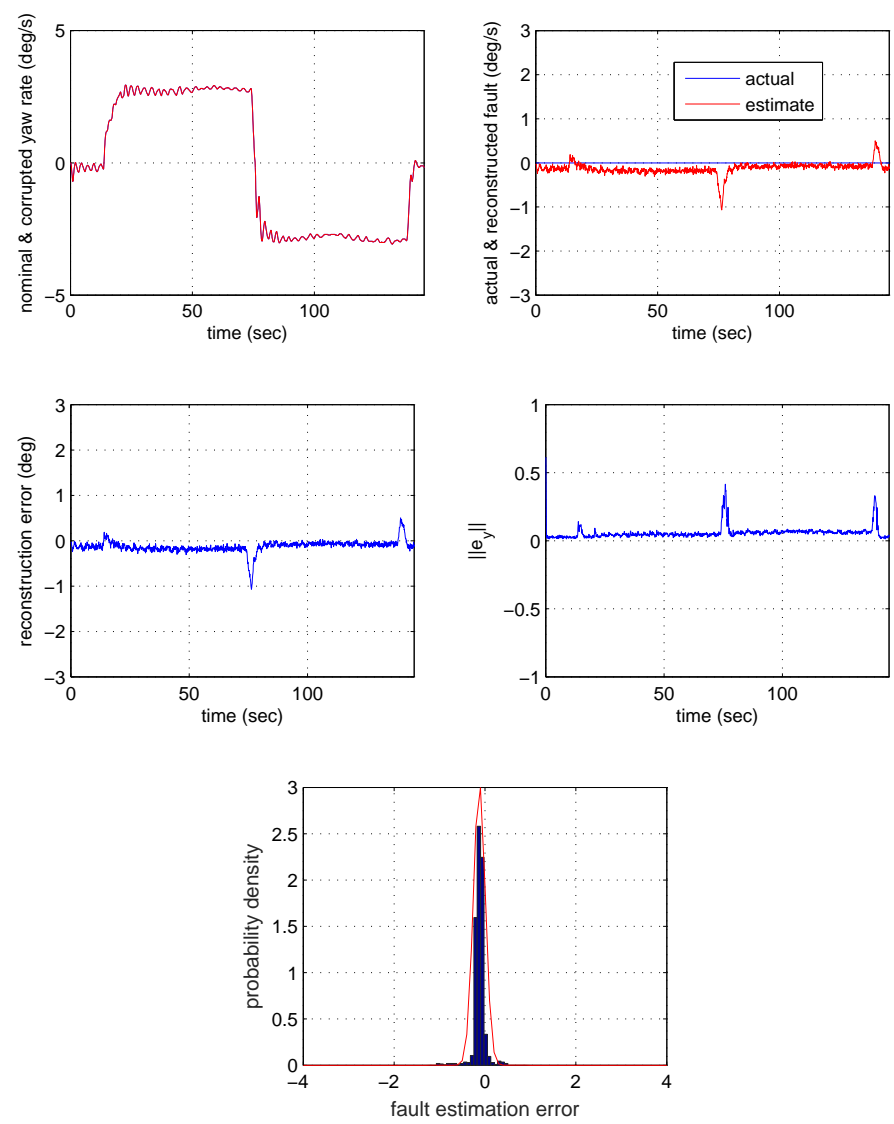

(b) Observer performance

Fig. 3. Fault-free case without winds: commands and observer performance

\section{ACKNOWLEDGEMENTS}

This research has received funding from the H2020 innovation programme under grant agreement No. 690811 , and from the Japan New Energy and Industrial Technology Development Organization under grant agreement No. 062800, as the research project entitled Validation of Integrated Safety-enhanced Intelligent flight cONtrol (VISION)'.

\section{REFERENCES}

[1] C. Edwards, T. Lombaerts, and H. Smaili, Fault tolerant flight control: A benchmark challenge. Springer, 2010.

[2] K. Masui and Y. Tsukano, "Development of a new in-flight simulator MuPAL- $\alpha$," AIAA paper 2000-4574, Aug. 2000.

[3] C. Edwards, S. K. Spurgeon, and R. J. Patton, "Sliding mode observers for fault detection and isolation," Automatica, vol. 36(4), pp. 541-553, 2000.

[4] H. K. Khalil and L. Praly, "High-gain observers in nonlinear feedback control," Int. J. Robust Nonlinear Control, vol. 24, pp. 993-1015, 2014.

[5] J. Stoustrup and H. H. Niemann, "Fault estimation - a standard problem approach," International Journal of Robust and Nonlinear Control, vol. 12, pp. 649-673, 2002. 

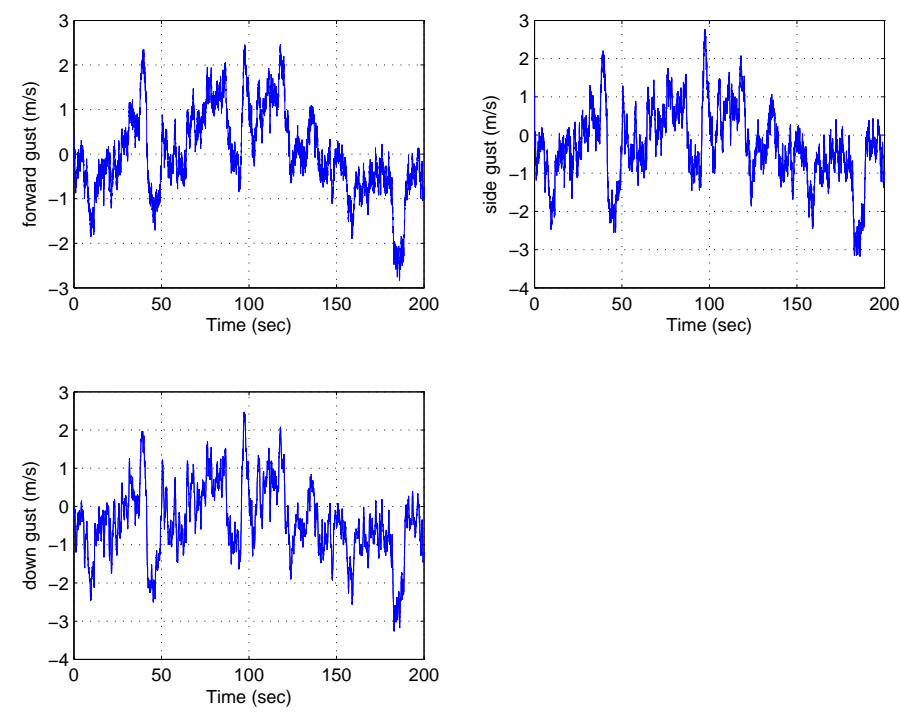

Fig. 4. Injected winds during HIL simulation

[6] B. Walcott, M. Corless, and S. Zak, "Comparative study of non-linear state-observation techniques," International Journal of Control, vol. 45, pp. 2109-2132, 1987.

[7] C. Edwards and C. P. Tan, "A comparison of sliding mode and unknown input observers for fault reconstruction," European Journal of Control, vol. 12, pp. 245 - 260, 2006.

[8] — "Sensor fault tolerant control using sliding mode observers," Control Eng. Pract., vol. 14, pp. 897-908, 2006.

[9] A. Marcos and M. Sato, "Flight testing of an structured H-infinity controller: An EU-Japan collaborative experience," in IEEE CCTA, 2017, pp. 2132-2137.

[10] G. Hardier, G. Ferreres, and M. Sato, "Design and flight testing of an adaptive gain-scheduled controller using on-line model estimation," in IEEE CCTA, 2018.

[11] H. Alwi, C. Edwards, and C. P. Tan, Fault Detection and Fault-Tolerant Control Using Sliding Modes. Springer, 2011.

[12] G.Strang, Linear Algebra and Its Applications, 5th ed. Wellesley-Cambridge Press and SIAM, 2016.

[13] V. Utkin, Sliding modes in control and optimization. Springer, 1992.

[14] M. Sato and A. Satoh, "Simultaneous realization of handling and gust responses: In-flight simulator controller design," Journal of Guidance, Control and Dynamics, vol. 31, 2008.

[15] — - "Flight control experiment of multipurpose-aviation-laboratory- $\alpha$ in-flight simulator," Journal of Guidance, Control, and Dynamics, vol. 34, 2011.

[16] S. Suzuki and A. Yanagida, "Research and development for fault tolerant flight control system - part 1. intelligent flight control system," in 26th International Congress of The Aeronautical Sciences, 2008.

[17] K. Masui, H. Tomita, and A. Yanagida, "Research and development for fault tolerant flight control system part 2 flight experiments," in 26th International Congress of the Aeronautical Sciences, 2008.

[18] D. Tokunaga, K. Masui, and S. Suzukia, "Flight evaluation of fault-tolerant control system using simple adaptive control method," in Asia-Pacific International Symposium on Aerospace Technology, APISAT2014, 2014.

[19] L. Chen, H. Alwi, C. Edwards, and M. Sato, "Flight evaluation of an LPV sliding mode controller with online control allocation," in IEEE CDC, 2017, pp. 3928-3933. 

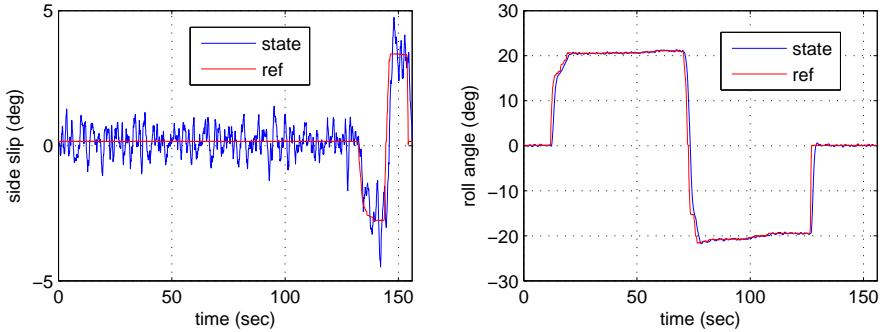

(a) Commands created by the evaluation pilot
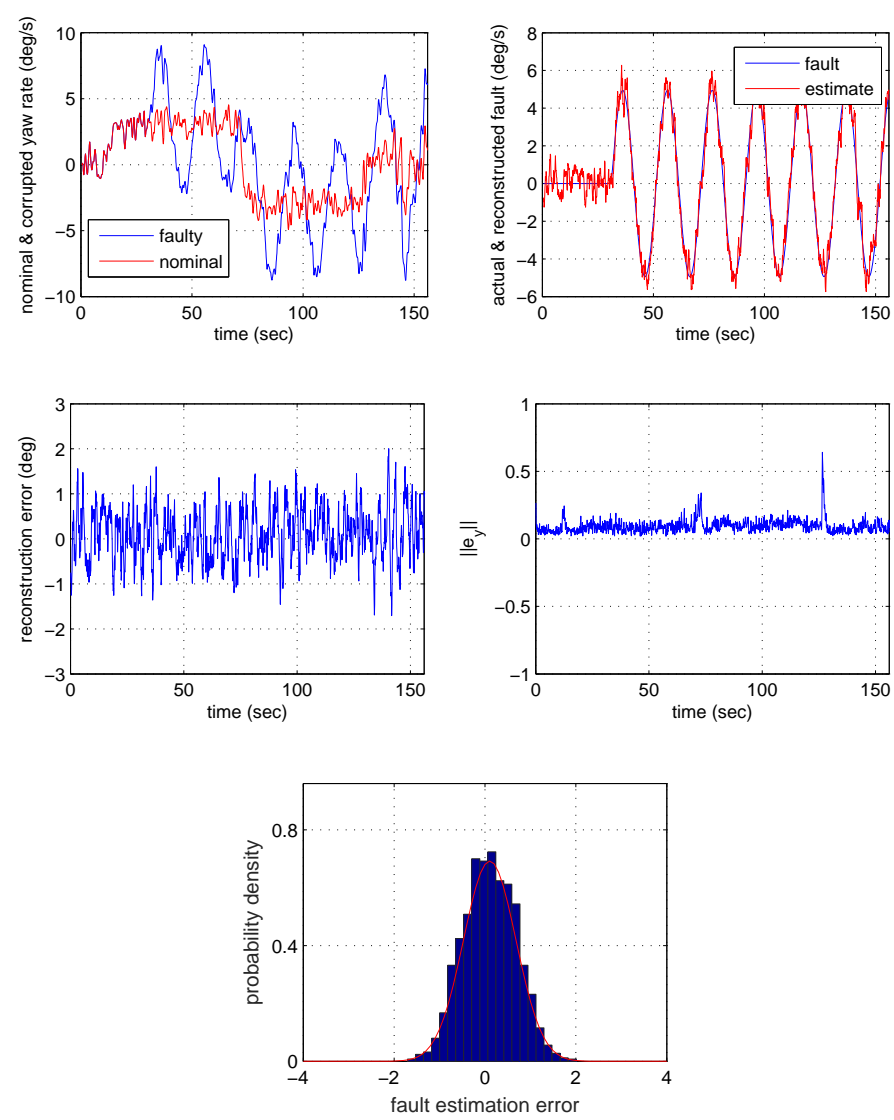

(b) Observer performance

Fig. 5. Sine wave fault case: commands and observer performance 